\title{
Effect of early identification on language acquisition in hearing impaired, cochlear implanted children
}

\author{
L C Seneviratne ${ }^{1}$ \\ Sri Lanka Journal of Child Health, 2013; 42: 87-89
}

\begin{abstract}
Objective: To determine the importance of early identification and intervention for hearing impairment towards the efficacy of a cochlear implant (CI) regarding language development

Method: A qualitative, observational study was carried out in Sri Lanka with regard to the hearing impaired (HI) children who underwent cochlear implants. They were recruited with the permission of their parents. The sample of HI children was obtained from places where hearing aids and cochlear implants were being sold in Sri Lanka and from the hospitals where they underwent cochlear implant surgery. Data were collected through direct observation as and when these HI cochlear implanted children showed changes in the process of language acquisition. Focus group discussions with ENT surgeons, audiologists, speech therapists and parents of these HI children were helpful in collecting data.
\end{abstract}

Results: Twelve HI children were recruited into this study 6 of them being prelingual $\mathrm{HI}$ and the others postlingual HI. Irrespective of being prelingual HI or postlingual $\mathrm{HI}$, the 8 who received the implant early in their life showed better language proficiency.

Conclusion: Early identification of hearing impairments in children and early intervention with cochlear implant lead to better language proficiency.

(Key words: Cochlear implant; hearing impairment; children)

\section{Introduction}

Hearing impairment is a disability which can be classified as prelingual hearing impairment and postlingual hearing impairment ${ }^{1}$. A prelingual

${ }^{1}$ Visiting Lecturer in English, English Language
Teaching Unit, University of Colombo

(Received on 20 July 2012: Accepted after revision on 24 August 2012) hearing impaired (HI) individual is someone whose hearing loss occurred before they began to speak, whereas a postlingual HI individual loses his/her hearing after he/she has learned to speak ${ }^{1}$. Hearing impairment could also be classified as mild, moderate, severe and profound hearing losses ${ }^{1}$. The second classification is based on the range of residuals an individual can grasp. Once individuals are found to have severe to profound hearing loss they should be supported by a cochlear implant as their auditory levels cannot be further supported by normal hearing aids.

A cochlear implant (CI) is a small, complex electronic device that can help provide a sense of sound to a person who is profoundly deaf or severely hard-of-hearing ${ }^{2}$. The implant consists of an external portion that sits behind the ear and a second portion that is surgically placed under the skin. An implant has the following parts: A microphone, which picks up sound from the environment, a speech processor, which selects and arranges sounds picked up by the microphone, a transmitter and receiver/stimulator which receive signals from the speech processor and convert them into electric impulses and an electrode array, which is a group of electrodes that collects the impulses from the stimulator and sends them to different regions of the auditory nerve ${ }^{2}$. An implant does not restore normal hearing but can give a deaf person useful representation of environmental sounds and help him or her understand speech ${ }^{2}$.

\section{Objective}

To determine the importance of early identification and intervention of hearing impairment towards the efficacy of a cochlear implant regarding language development

\section{Method}

A qualitative, observational study was carried out in Sri Lanka with regard to HI children who underwent cochlear implants, irrespective of the districts, as it took place according to the places where these hearing impaired, cochlear implanted children lived. 
Furthermore, they were recruited with the permission of their parents after meeting them. The researcher was able to find the sample of hearing impaired children from the places where hearing aids and cochlear implants were being sold in Sri Lanka and from the hospitals where they underwent CI surgery.

Data were collected through direct observation as and when these HI cochlear implanted children showed changes in the process of language acquisition. Moreover, focus group discussions with ENT surgeons, audiologists, speech therapists and especially with the parents of these HI children were helpful in collecting data.

\section{Results}

Twelve HI children, who had undergone cochlear implant surgery contemporarily, were recruited into the study. This included 6 prelingual and 6 postlingual HI children. All 6 postlingual hearing impairments were diagnosed as after-effects of meningitis and 4 out of 6 were from the Kandy district. At the same time the sample consists of 8 children whose impairment had been detected early.

The $8 \mathrm{HI}$ children, whose hearing impairment had been detected early, irrespective of them being prelingual HI or postlingual HI, were able to develop their first language faster in comparison to the other 4 HI children. All 12 children had to be trained to discriminate the surrounding sounds from other noises. Subsequently, they were given proper guidance as to how they should produce sounds, initially onomatopoeic sounds (which means the words that imitate the sound they denote). These activities were more successfully grasped by those 8 children who were detected early than the other 4 children who were deprived of auditory input for a considerable period of time. There were 2 prelingual $\mathrm{HI}$ children who underwent surgery at the age of 2 years and $2 \frac{1 / 4}{4}$ years of age respectively who demonstrated a rapid development in their language perception as well as in their language production in comparison to the other 6 who underwent the cochlear implant (CI) surgery later. When these 2 children started producing one syllabic words, the other 6 were still producing onomatopoeic sounds connected with their day to day life. However, the children who underwent CI surgery late in their life were ahead in comprehending what was being said by the others compared to the 2 small children who underwent the surgery early.

It was noticed that 2 postlingual HI children, whose hearing impairment was diagnosed later, found it very difficult to produce language even though they were quite familiar with it for some time. Furthermore, it was noted that even the other 4 post lingual HI children were somewhat behind in the rate of language production in comparison to the prelingual $\mathrm{HI}$ children who underwent CI surgery. It was found that the 2 prelingual $\mathrm{HI}$ children who underwent CI surgery when they were 2 years and $2 \frac{1}{4}$ years of age, showed a more rapid improvement in language perception and production than the other 4 prelingually $\mathrm{HI}$ children who received a CI later in their life from $3 \frac{1}{2}$ to 5 years of age.. The postlingual hearing impairment of these 6 children had occurred when they were between 4 and 5 years of age after meningitis. Similarly, when the 6 postlingual HI children were observed for their language proficiency, they too showed similar results as 4 of them who received the CI early had better language proficiency compared to the other 2 postlingual hearing impaired $\mathrm{CI}$ children. The 2 postlingual hearing impaired CI children were comfortable with other modes of non-verbal communication like sign language and lip reading. The parents of these $12 \mathrm{HI}$ children were given proper instructions regarding handling of the CI device. However, 3 parents complained that they were unable to understand all the necessary steps which should be followed when handling their child's CI device and it was noticed that the children of these parents have found it more difficult to produce language in comparison to other CI children.

\section{Discussion}

Once a child is diagnosed with hearing impairment he or she should be taken to a paediatric ENT surgeon where he/she could get all the necessary medical testing and audiological testing including different types of audiograms which will give specific details about the type of hearing impairment. Accordingly, the parents will be informed of the type of hearing impairment and the remedy for it, whether it is going to be just a pair of conventional hearing aids or a CI.

A CI is different from a hearing aid. Hearing aids amplify sounds so that they may be detected by damaged ears. Cochlear implants bypass damaged portions of the ear and directly stimulate the auditory nerve. Signals generated by the implant are sent by way of the auditory nerve to the brain, which recognizes the signals as sound ${ }^{2}$. Hearing through a $\mathrm{CI}$ is different from normal hearing and takes time to learn or relearn ${ }^{2}$. Cochlear implantation is a collaborative effort involving patients, families, schools, audiologists, speech/hearing therapists and 
surgeons $^{3}$. Normally, as preoperative expectation affects the patient's postoperative satisfaction and use of implant, all patients and families require attention and counseling from an implant team before they undergo cochlear implantation.

The ability to speak is closely related to the ability to hear. If the child is not able to hear, then the child will have difficulty learning how to speak correctly. Hearing provides feedback which is used by the child to correct or improve his/her speech production skills ${ }^{4}$. Auditory feedback is therefore very important for learning how to speak, and cochlear implants can provide that. Research has shown that the intelligibility of speech produced by children with cochlear implants improves over time ${ }^{4,5}$. Parents, who were able to identify the deterioration in hearing in their children early were able to seek immediate treatment and consequently even 4 postlingual $\mathrm{HI}$ children were able to gain proper auditory input somewhat similar to what they had earlier within a considerable period of time. However, the other 2 post lingual HI children who were not identified for their deteriorating hearing loss, were left for some time without proper auditory input and due to that faced a tendency to forget the language they were using for a few years of time. Thus these 2 children were more comfortable with other modes of nonverbal communication like sign language and lip reading and these methods sometimes fossilized within them and it was later found very difficult to change their ways of communication even with proper auditory input.

After 1-4 weeks of healing, the implant is "activated" by connecting an external sound processor to the internal device via a magnet. Initial results vary widely, and post-implantation therapy is required as well as time for the brain to adapt to hearing new sounds ${ }^{5}$. In the case of congenitally deaf children, audiological training and speech therapy typically continue for years, though infants can become age appropriate-able to speak and understand at the same level as a hearing child of the same age ${ }^{5}$. The participation of the child's family in working on spoken language development is considered to be even more important than therapy, because the family can aid development by participating actively - and continually_-in the child's therapy, making hearing and listening interesting, talking about objects and actions, and encouraging the child to make sounds and form words ${ }^{6}$.

\section{Conclusion}

This study shows that early identification of hearing impairment in children and early intervention with cochlear implants lead to better language proficiency.

\section{References}

1. Hearing impairment. Available from: http://encyclopedia.thefreedictionary.com/Hearin g+impairment

2. National Institute on Deafness and other Communication Disorders (NIDCD). Cochlear implants. Available from:

file://C:/Users/techno/Downloads/Cochlear\%20I mplants/Cochlear\%20Implants.htm

3. Isaacson B. Indications for cochlear implants. Available from:

file:///C:/Users/techno/Downloads/Cochlear\%20I mplants/857164-overview.htm

4. Tobey E. Speech production. In: R. Tyler, editor. Cochlear Implants: Audiological Foundations Singular Publishing Group, Inc: 1993. p. 257316 ,

5. Osberger M, Robbins A, Todd S, Riley A, Miyamoto R. Speech production skills of children with multichannel cochlear implants. In: Hochmair-Desoyer I, Hochmair E, editors. Advances in cochlear implants. Vienna: Manz, 1994; p. 503-7.

6. Cochlear implant. Available from: file://C:/Users/techno/Downloads/Cochlear\%20I mplants/Cochlear_implant.htm 